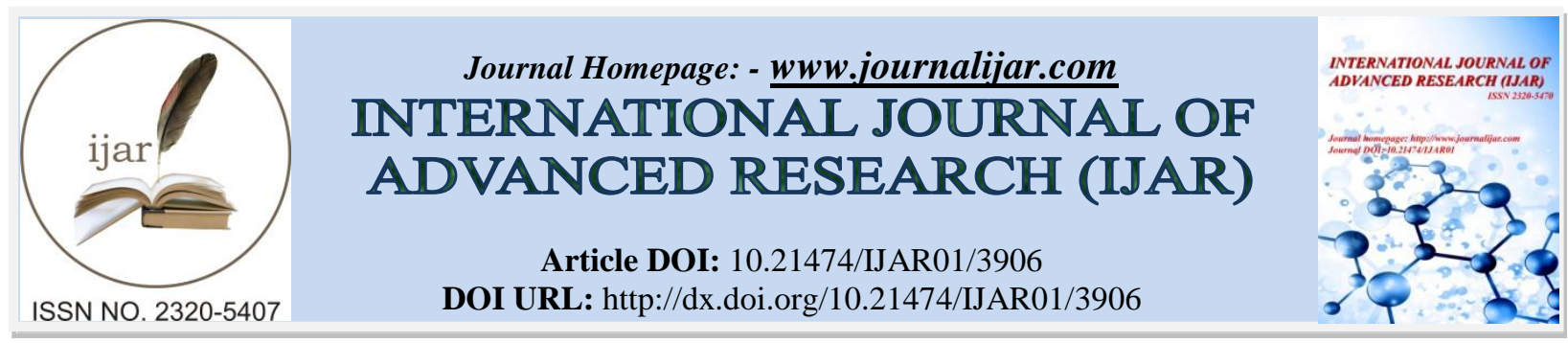

RESEARCH ARTICLE

\title{
APEXOGENESIS WITH DIRECT PULP CAPPING USING A NEW BIOACTIVE MATERIAL IN AN IMMATURE LOWER INCISOR - A CASE REPORT
}

Dr. P. V. Sreedevi, Dr.Syed Inayathulla, Dr.B.G.Yogesh, Dr.Arun J Kumar, Dr.Ashwini and Dr.P.Sheshadri.

\section{Manuscript Info}

Manuscript History

Received: 08 February 2017

Final Accepted: 05 March 2017

Published: April 2017

Key words:-

Biodentine, immature tooth, pulp

capping, apexogenesis

\begin{abstract}
Coronal fractures of the anterior teeth are a common form of dental trauma that mainly affects children and adolescents. Preservation of the pulp in a traumatized immature fractured incisor tooth is of prime importance in order to achieve apexogenesis, a natural apical closure. Apexogenesis after traumatic exposure in vital young permanent teeth can be accomplished by implementing an appropriate vital pulp therapy such as pulp capping (direct or indirect) depending on time interval between the trauma and treatment, degree of root development, and size of the pulp exposure. Pulpal protection by a bioactive material may provide optimum marginal seal in preventing microleakage. This case report presents an 8 year old male patient diagnosed with Ellis Class III fracture of immature lower anterior tooth 31 , exposed pulp was sealed using a new bioactive material Biodentine to protect the pulp and stimulate apexogenesis. The fractured segment was reattached for optimum esthetics. The patient was followed up for 1, 3, 6 and 12 months, which revealed continued apical closure and maintenance of pulp vitality. The patient remained asymptomatic. This case report provides evidence for the potential use of biodentine as an effective pulp capping material.
\end{abstract}

Copy Right, IJAR, 2017,. All rights reserved.

\section{Introduction:-}

Coronal factures of the anterior teeth are a common form of dental trauma that mainly affects children and adolescents ${ }^{\mathbf{1 , 2}}$. The majority of dental injuries involve the anterior teeth. Direct pulp capping involves the application of a dental material to seal communications between the exposed pulp and the oral cavity (mechanical and carious pulp exposures) in an attempt to act as a barrier, protect the dental pulp complex and preserve its vitality ${ }^{3}$. Inducing hard tissue formation by pulp cells is an ultimate goal for a capping material ${ }^{4}$.

Biocompatible materials such as calcium hydroxide and more recently mineral trioxide aggregate (MTA) are commonly used for pulp capping ${ }^{5,6}$ Calcium hydroxide is the most reliable material for direct and indirect pulp capping and serves as the "gold standard" . Calcium hydroxide does not prevent microleakage in the long run. This may cause secondary inflammation of the pulp tissue and result in loss of tooth vitality. In addition, the high $\mathrm{pH}(12.5)$ of calcium hydroxide suspensions causes liquefaction necrosis at the surface of the pulp tissue ${ }^{\mathbf{8}}$. Nevertheless, calcium hydroxide has some drawbacks. Poor bonding to dentin, material reabsorption, high solubility and mechanical instability are among them. 
Biodentine was developed aiming to improve MTA drawbacks such as its difficult handling property and long setting time. Biodentine is new bioactive cement with dentin like mechanical properties that can be used as a dentin substitute on crowns and roots. It has a positive effect on vital pulp cells and stimulates tertiary dentin formation.

This case report is a case of Ellis class III tooth fracture that was successfully treated using biodentine.

\section{Case Report:-}

An 8 year old male patient reported to the department of Conservative Dentistry and Endodontics, with a complaint presenting crown fracture of left mandibular central incisor (Fig 1) due to sports related trauma four hours back. On clinical examination, the tooth displayed an Ellis Class III fracture. The patient gave a history of sensitivity to cold after fracture, indicating that the pulp was vital. The tooth was non tender and showed no mobility. The exposed pulp did not show evidence of haemorrhage. The patient also reported with the fractured tooth fragment.

Radiographic evaluation (Fig 2) was performed to exclude underlying periapical pathology and root fractures.

The fractured tooth fragment was checked for its integrity and was cleaned with sodium hypochlorite and then kept in normal saline. Small piece of fragment was not traceable, composite treatment was planned to replace the missing portion of tooth fragment. Prior to the execution of the proposed treatment, a consent form duly signed by patient's parents was taken.

The tooth was anesthetized and was isolated using rubber dam. A groove was made on tooth for material adaptation to the tooth surface, pulp tissue was irrigated with normal saline. Biodentine was mixed according to the manufacturer's instructions (Septodont) and was gently placed in the cavity (Fig3). After the initial setting of biodentine, a bevel was made on the facial and lingual surfaces of the tooth and the fractured fragment to prepare them for reattachment. The surfaces were etched using 35\% phosphoric acid and bonding agent (Adper, 3M ESPE) was applied and light cured for 20 seconds. A layer of flowable composite (Tetric Flow, Ivoclar) was applied on both the surfaces and was approximated to the original tooth contour and light cured for $40 \mathrm{~s}$. The missing portion of fragment was compensated with composite resin. The tooth was finished and polished (Fig4).

The patient was recalled on next day for follow up. On clinical examination the tooth was sound and the patient was asymptomatic. Periodic recall and radiographs were analyzed after 1 month (Fig 5), 3 months (Fig 6), 6 months (Fig 7) and 12 months (Fig8). The patient remained asymptomatic and radiographs displayed continued root formation.

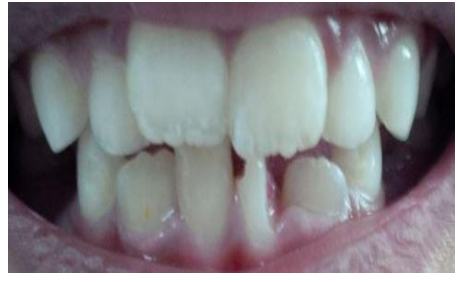

Fig 1:- Preoperative.

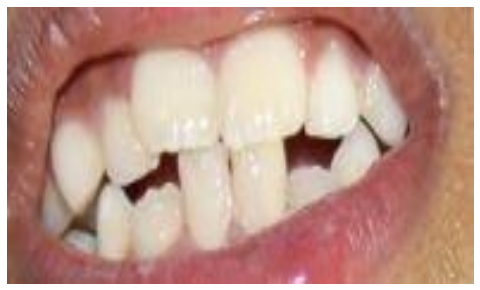

Fig 4;- Post Operative

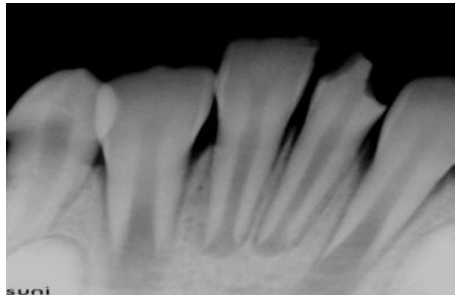

Fig 2:- Pre-Operative Radiograph

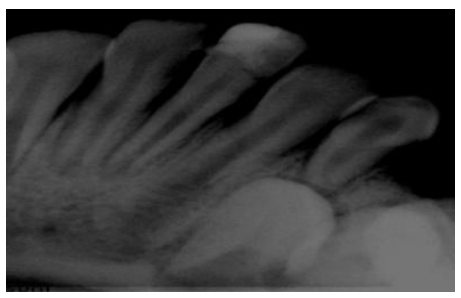

Fig 5:- 1 Month Follow Up

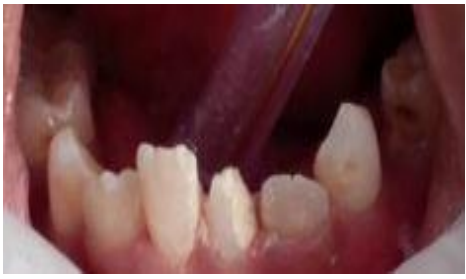

Fig 3:- Biodentin Placement

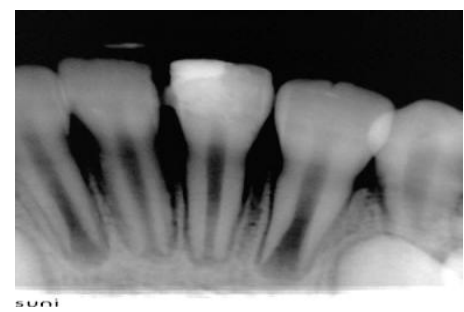

Fig 6:- 3 Months Follow Up 


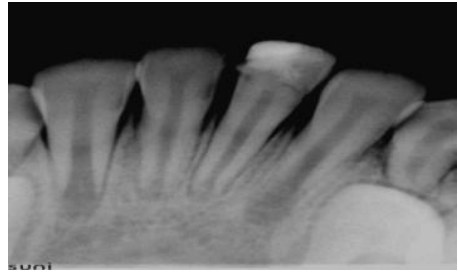

Fig 7:- 6 Months Follow Up

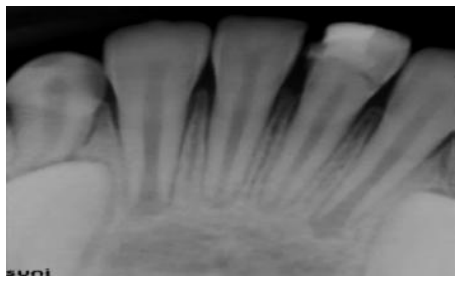

Fig 8:- 1 year follow up

\section{Discussion:-}

Bioactive cement, Biodentine (Septodont), was launched in the dental market as a dentin substitute alternative to calcium hydroxide and MTA. Biodentine consists of powder in a capsule and liquid in a pipette. The powder mainly contains tricalcium and dicalcium silicate as well as calcium carbonate. Zirconium dioxide is the contrast medium. The liquid consists of calcium chloride in aqueous solution with an admixture of polycarboxylate. The powder is mixed with the liquid in a capsule in the triturator for 30s. Once mixed, biodentine sets in about $12 \mathrm{~min}$. During the setting of the cement, calcium hydroxide is formed. The consistency of biodentine is similar to that of phosphate cement ${ }^{8}$.

Biodentine preserves pulp vitality and promotes its healing process. Laurent et al tested a new $\mathrm{Ca}_{3} \mathrm{SiO}_{5}-$ based material to evaluate its genotoxicity, cytoxicity and effects on the target cells specific function. The study concluded that the biodentine material is biocompatible ${ }^{10}$.

Apexogenesis is a treatment to preserve vital pulp tissue in the apical part of root canal to complete formation of root apex. In the present case, Biodentine was selected over calcium hydroxide and MTA. In pulp capping, the benefits of biodentine over calcium hydroxide are: mechanically strong, less soluble and produces tighter seals ${ }^{11,12,13}$. Compared to MTA, biodentine displays ease of handling and decreased setting time. It is sufficiently stable so that it can be used both for pulp protection and temporary fillings ${ }^{8}$. Biodentine exhibits better mechanical properties than MTA. Biodentine does not require a two-step restoration procedure as in the case of MTA. As the setting is faster, there is a lower risk of bacterial contamination than with MTA. Apart from the choice of the agent used for pulp capping, the other factors that need to be considered are the clinical status of the pulp and the microbial contamination of the pulp at the site of injury.

Reattached fragment to a great extent restores esthetics as it uses the original tooth's shape, color, translucence and surface structure. ${ }^{14}$. Reis et $\mathrm{al}^{15}$ have shown that a simple reattachment with no further preparation of the fragment of tooth was able to restore only $37.1 \%$ of the intact tooth's fracture resistance, whereas a buccal chamfer recovered $60.6 \%$ of that fracture resistance; bonding with an over contour and placement of an internal groove nearly restored the intact tooth fracture strength, recovering $97.2 \%$ and $90.5 \%$ of it, respectively.

During follow-up of traumatized teeth reported at different time periods, no tooth sensitivity or pain was reported; also, neither clinical symptoms nor radiographic defects were present. Clinical and radiographic examination showed continual apexogenesis with no periodontal or periapical pathology and the restorations were functionally acceptable and aesthetically gratifying.

\section{Conclusion:-}

The good handling properties of biodentine associated with its favorable biological, mechanical and physical properties indicate that it can be used efficiently in clinical practice as a pulp capping material alternative to calcium hydroxide and MTA. However, long term evaluation in clinical situations is required for further inferences. 


\section{References:-}

1. Dietschi D, Jacoby T, Dietchi JM, Schartz JP. Treatment of traumatic injuries in the front teeth: restorative aspects in crown fractures. Pract Periodontics Aesthet Dent 2000; 12(8):751-8.

2. Hamilton FA, Hill FJ, Holloway PJ. An investigation of dento-alveolar trauma and its treatment in an adolescent population. Part 1: the prevalence and incidence of injuries and adequacy of treatment received. Br Dent J 1997; 182(3):91-5.

3. European Society of Endodontology. Quality guidelines for endodontic treatment: consensus report of the European Society of Endodontology. Int Endod J.2006; 39(12):921-930.

4. Obeid M, SaberSel D, Ismael Ael D, Hassanien E. Mesenchymal stem cells promote hard-tissue repair after direct pulp capping. J Endod.2013;39(5):626-31.

5. Dominguez MS, Witherspoon DE, Gutmann JL, Opperman LA. Histological and scanning electron microscopy assessment of various vital pulp-therapy materials. J Endod.2003;29(5):324-33.

6. Camilleri J, Pitt-Ford TR. Mineral trioxide aggregate: a review of the constituents and biological properties of the material. Int Endod J. 2006; 39(10):747-54.

7. Horsted-Bindslev P, Vilkinis V, Sidlauskas A. Direct pulp capping of human pulps with a dentin bonding system or with calcium hydroxide cement. Oral Surgery Oral Medicine Oral Pathology Oral Radiology \& Endodontology. 2009; 96:591-600.

8. Bhat SS, Hegde SK, Adhikari F, Bhat VS. Direct pulp capping in an immature incisor using a new bioactive material. Contemp Clin Dent 2014; 5(3):393-6.

9. Hauman $\mathrm{CH}$, Love RM. Biocompatibility of dental materials used in contemporary endodontic therapy: a review. Part 2. Root canal-filling materials. Int Endod J 2003; 36:147-60.

10. Priyalakshmi. S, Manish R. Review on Biodentine - A Bioactive Dentin Substitute. IOSR-JDMS; 2014 ;13(1);13-17

11. Foreman PC, Barnes IE. Review of calcium hydroxide. Int Endod J 1990; 23(6):283-97.

12. Aljandan B, AlHassan H, Saghah A, Rasheed M, Ali AA. The effectiveness of using different pulp capping agents on the healing response of the pulp. Indian J Dent Res 2012;23(5):633-7.

13. Han L, Okiji T. Bioactivity evaluation of three calcium silicate- based endodontic materials. Int Endod J 2013; 46:808:14.

14. De Lourdes Rodrigues Accorinte M, Reis A, DouradoLoguercio A, Cavalcanti de Araujo V, Muench A. Influence of rubber dam isolation on human pulp responses after capping with calcium hydroxide and an adhesive system. Quintessence Int 2006; 37(3):205-12.

15. Reis A, Francci C, Loguercio AD, et al. Reattachment of anterior fractured teeth: fracture strength using different technique. Oper Dent 2001; 26(3):287-94. 